\title{
Hemoperitoneo y muerte por rotura de metástasis hepatoesplénicas de tumor testicular
}

\author{
Pastor Navarro H, Donate Moreno MJ, Ruiz Mondéjar R, Pastor Guzmán JM, \\ Salinas Sánchez AS, Virseda Rodríguez JA.
}

Servicio de Urología. Complejo Hospitalario y Universitario de Albacete.

Actas Urol Esp. 2007;31(10):1175-1178

\section{RESUMEN}

HEMOPERITONEO Y MUERTE POR ROTURA DE METÁSTASIS HEPATOESPLÉNICAS

DE TUMOR TESTICULAR

Presentamos un caso de tumor germinal mixto de testículo, en estadio III-C (AJCC-UICC), con evolución letal, por hemoperitoneo masivo por rotura de metástasis hepáticas y esplénicas.

Palabras clave: Tumor de testículo. Cáncer de testículo. Tumor de células germinales. Hemoperitoneo.

\section{ABSTRACT \\ HEMOPERITONEUM AND DEATH DUE TO HEPATOESPLENIC METHASTASIS RUPTURE IN A TESTICULAR TUMOR}

We present a case of a mix germinal testicular tumor,in III-C (AJCC-UICC) stage, at a terminal phase due to massive methastasis rupture at hepatic and splenic methastasis locations.

Keywords: Testicular tumor. Testicular cancer.Germinal cell tumor. Hemoperitoneum.

$\mathrm{E}$ diagnóstico de una tumoración testicular iene gran repercusión debido al impacto que ocasiona sobre el paciente por la edad de presentación, tratamiento necesario y sus implicaciones en cuanto a fertilidad. Se estima que alrededor de un $20 \%$ de casos presenta metástasis al diagnóstico, siendo muy rara la evolución letal de la enfermedad por hemorragia intraabdominal por rotura de metástasis hepato-esplénicas

\section{CASO CLÍNICO}

Paciente de 15 años sin antecedentes previos de interés. Fue asistido en urgencias por presentar vómitos y dolor epigástrico de tres días de evolución, y refería la pérdida de $7 \mathrm{~kg}$ de peso en los dos meses anteriores. A la exploración física se apreció moderada palidez mucocutánea, hepatoesplenomegalia de dos traveses de dedo y dolor epigástrico a la palpación profunda. La exploración escrotal demostró un teste izquierdo aumentado de volumen (tres o cuatro veces más de lo normal) de consistencia dura, superficie irregular y no doloro- so. Reinterrogado el paciente, nos confirmó, que desde hacía aproximadamente un año había notado un aumento progresivo e indoloro del teste.

En la analítica de urgencias únicamente destacó un hematocrito de 35\%, una hemoglobina de 11,3 gr/dl y una LDH de $2802 \mathrm{U} / \mathrm{L}$.

La ecografía abdominal y testicular demostró la existencia de un teste izquierdo muy aumentado de tamaño (Fig. 1) de ecogeneicidad heterogénea, con focos de necrosis y áreas líquidas; y a nivel hepático amplias imágenes sugestivas de metástasis (Fig. 2).

La radiología de tórax evidenció la existencia de metástasis pulmonares múltiples y discreto derrame pleural, que tras toracocentesis se obtuvo líquido pleural hemático.

Doce horas después del ingreso se realizó TAC toracoabdominal donde se demostró una extensa afectación metastásica, con nódulos pulmonares múltiples bilaterales, derrame pleural derecho (Fig. 3), adenopatías mediastínicas, retroperitoneales, hepatomegalia con afectación metastási- 


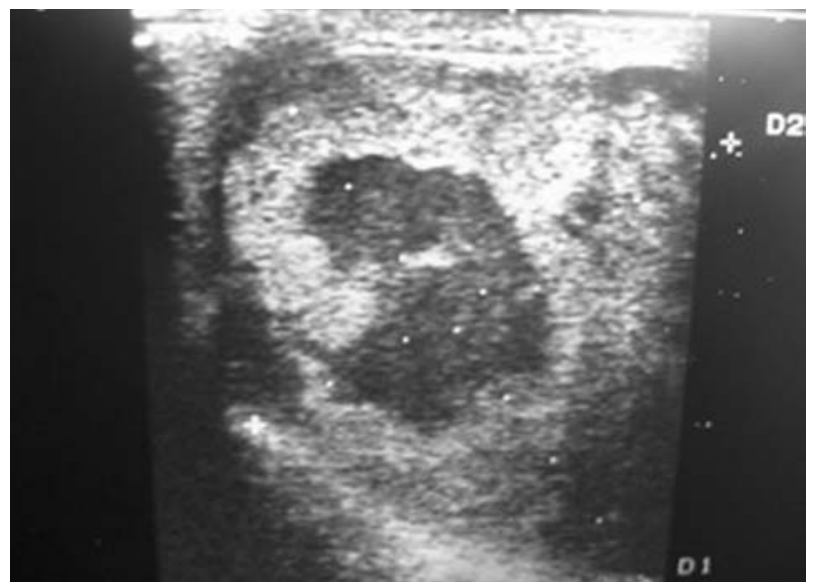

FIGURA 1

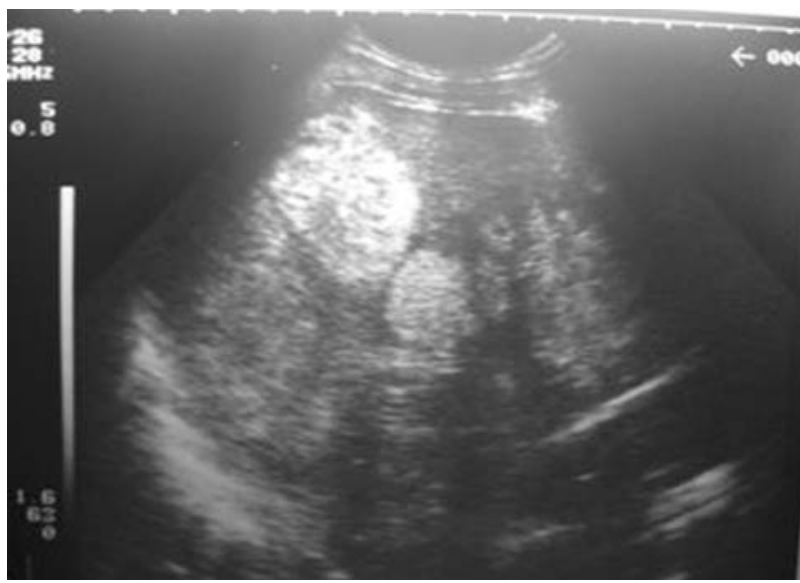

FIGURA 2

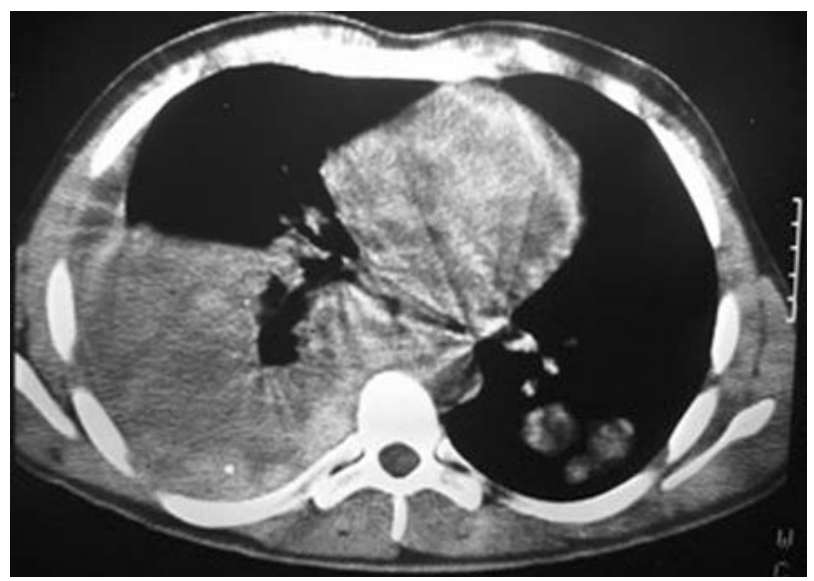

FIGURA 3

ca masiva, (Fig. 4 y 5) y posiblemente metástasis esplénicas y pancreáticas. No existía líquido libre intraperitoneal.

Se obtuvo muestras de sangre para marcadores testiculares: alfafetoproteína $15000 \mathrm{ng} / \mathrm{ml}$ y betaHCG de $200.000 \mathrm{mUI} / \mathrm{ml}$. y se programó orquiectomía para el día siguiente.

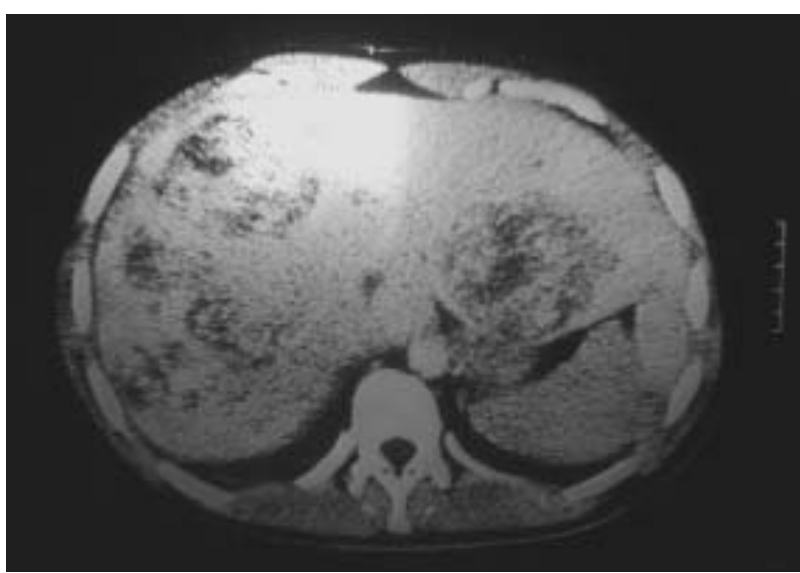

FIGURA 4

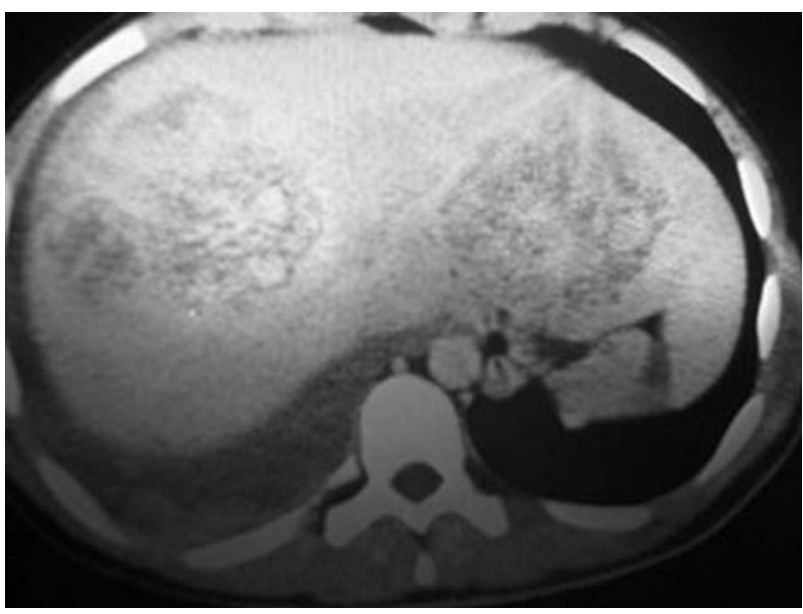

FIGURA 5

A las 36 horas del ingreso, se produjo shock hipovolémico de instauración rápida con dolor y abombamiento abdominal, que requirió laparotomía urgente tras ecografía abdominal en la que demostró una gran cantidad de líquido intraperitoneal. Durante la laparotomía se comprobó la existencia de afectación metastásica extensa en cola de páncreas, bazo e hígado con rotura de metástasis esplénicas y hepáticas y masivo hemoperitoneo por sangrado activo de las mismas y con hematoma difuso hepático. Se efectuó esplenectomía con sutura y taponamiento con surgicel de las áreas hepáticas sangrantes; al mismo tiempo se practicó orquiectomía radical izquierda.

La anatomopatológica macroscópica describe: "pieza de orquiectomía radical, que pesa 233 gr y mide 8x9x5 cm, acompañado de $7 \mathrm{~cm}$ de cordón; a los cortes seriados el parénquima testicular muestra un reemplazamiento casi total por una 
tumoración blanquecina, con áreas de necrosis y hemorragia que no parece rebasar las cubiertas testiculares".

La histopatología microscópica del testículo mostró una neoplasia maligna germinal mixta en la que se aprecian áreas de tipo carcinoma embrionario, tumor del seno endodérmico, coriocarcinoma y teratoma (Figs. 6 y 7).

El bazo media $15 \times 7 \times 3 \mathrm{~cm}$ y pesaba $180 \mathrm{gr}$, observándose en los cortes seriados seis formaciones nodulares, redondeadas, blanquecinas, con necrosis central. La microscopía confirma que las lesiones esplénicas son metástasis de la neoplasia testicular (Fig. 8).

Cuarenta y ocho horas después de la intervención el enfermo sufrió un nuevo cuadro de shock hipovolémico con signos evidentes de sangrado intraabdominal, por lo que se efectuó nueva laparotomía, verificándose la existencia de estallido hepático a nivel de lóbulo derecho e izquierdo, con hemorragia incoercible y deceso poco después.

\section{DISCUSION}

Los tumores testiculares son frecuentes en varones jóvenes siendo la primera enfermedad maligna en pacientes entre los 25 y los 35 años. Un $20 \%$ de los casos puede presentarse con metástasis en el momento del diagnóstico.

La hemorragia intraabdominal es poco frecuente, tanto en lesiones benignas como malignas, pero es mas habitual en lesiones primarias que en las metastásicas ${ }^{1}$. En varones jóvenes con abdomen agudo, la exploración escrotal no debe omitirse nunca.

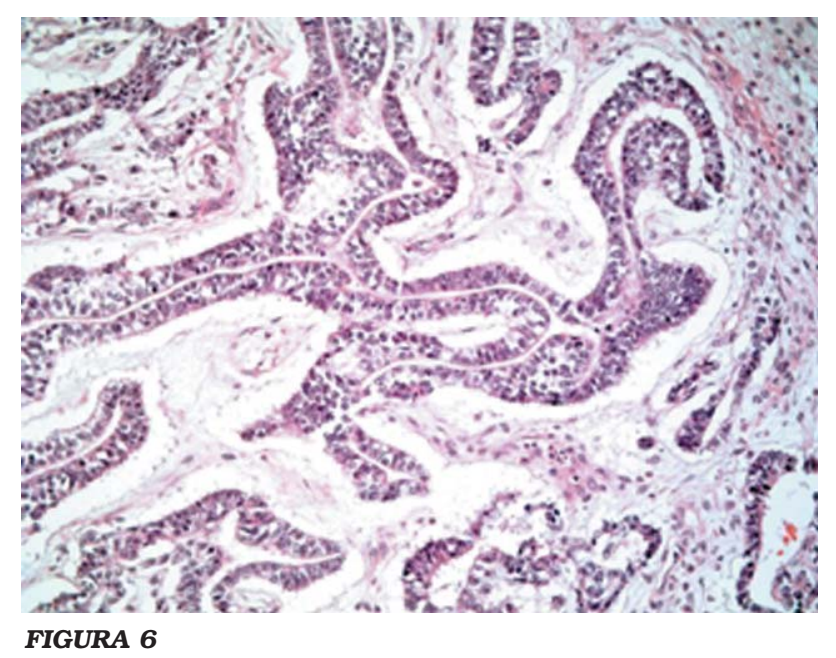

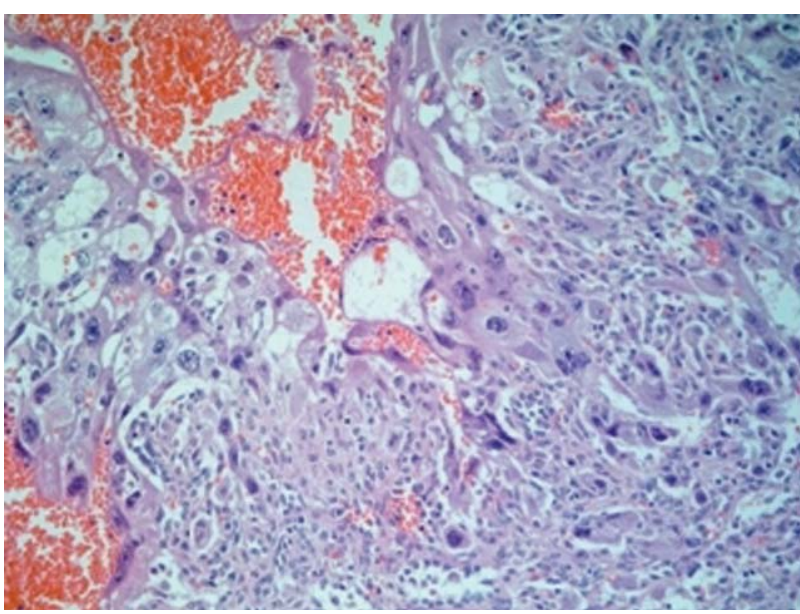

FIGURA 7

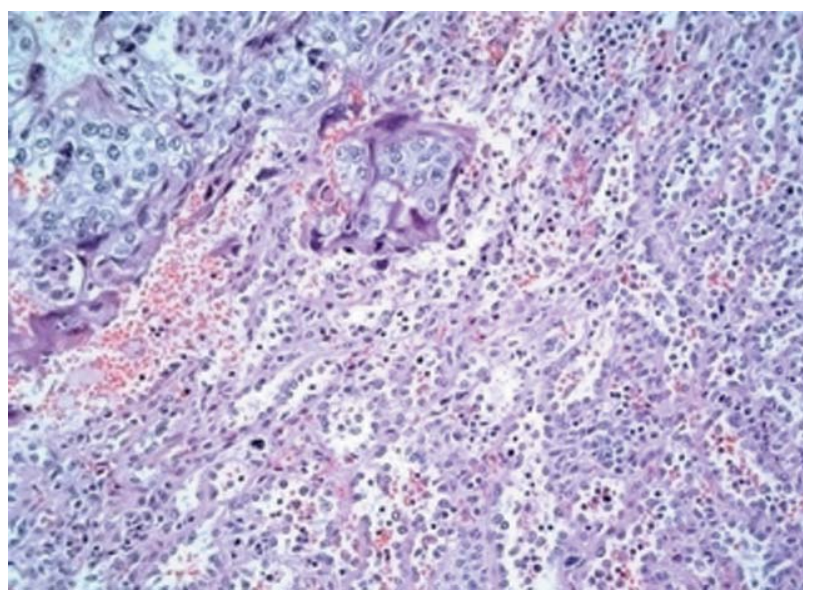

FIGURA 8

Los traumatismos y patologías ginecológicas no malignas suponen más del 90\% de las hemorragias abdominales. El hemoperitoneo en patologias malignas es más común en coriocarcinómas y seminómas de testículos criptorquídicos intraabdominales ${ }^{1-4}$, tumores de la granulosa ováricos y en el carcinoma primario y hemangioendoteliosarcoma hepáticos ${ }^{5-7}$. Algún caso se ha descrito de hemoperitoneo por rotura hepática con patología benigna como es en el adenoma hepatocelular ${ }^{6}$. Se han publicado algunos casos de hemorragia pélvica o retroperitoneal tras orquiectomía radical $^{8}$ por ligadura ineficaz y retracción de las venas del cordón. El hemoperitoneo por rotura hepática en hígado metastatizado por tumores testiculares, es mas frecuente cuando este es más agresivo, como ocurre en los coriocarcinomas, carcinomas embrionarios o en los mixtos con alguno de estos dos componentes ${ }^{5,7,9,10}$ como en el caso que presentamos. 
El hemoperitoneo se puede producir de forma espontánea o tras tratamiento quimioterápico. Ocurre en tumores con alta tasa de proliferación, en los que se produce necrosis espontánea, por afectación e invasión vascular ${ }^{5}$ o inducida por la quimioterapia, con la rotura y la hemorragia subsecuente $^{11}$.

El coriocarcinoma es el mas agresivo de los tumores de células germinales, conteniendo histológicamente cito y sincitiotrofoblasto; se disemina por vía linfática y sanguínea; responde bien a la quimioterapia aunque sea metastásico, con una tasa de supervivencia a los 5 años del 70$80 \%$, sin embargo la existencia de complicaciones hemorrágicas ensombrece el pronóstico ${ }^{12}$. Se caracteriza por la rápida proliferación, invasividad, gran vascularización y tendencia al sangrado como consecuencia de la necrosis tumoral ${ }^{10} \mathrm{y}$ alteraciones de la coagulación. Algunos factores de la coagulación (II,VII, X) y la vit K se sintetizan en el hígado, y cuando este es metastatizado, se puede inducir una coagulopatía de consumo (CID), por activación de los mismos, con tendencia secundaria a las hemorragias ${ }^{13}$. En el tracto genitourinario la CID puede asociarse al adenocarcinoma prostático metastásico, pero el coriocarcinoma es otro tumor potenciador de su aparición ${ }^{13}$. El masivo reemplazamiento de tejido hepático por tumor, asociado a deficiencias nutricionales, puede producir una depleción de factores de coagulacion ${ }^{7}$, que favorecerá también la aparición de hemorragias.

En los casos de muerte por coriocarcinoma testicular, la hemorragia esta implicada en el $44 \%$ de ellos como causa de la misma ${ }^{14}$.

\section{REFERENCIAS}

1. Watkins GL. Massive hemoperitoneum resulting from rupture of a seminoma in an undescended testicle. J Urol. 1970;103(4):447-448.

2. Wilson L. Rupture and hemorrhage of an intra-abdominal testicular tumor in a pseudohermaphrodite. West J Surg Obstet Gynecol 1961;69:312-314.
3. Küçük HF, Dalkiliç G, Kuro?lu E, Altunta? M, Bari?ik NO, Gülmen M. Massive bleeding caused by rupture of intraabdominal testicular seminoma: a case report. J Trauma. 2002;52(5):1000-1001.

4. Feldbaum DM, Goldstein S. Acute haemoperitoneum caused by intra-abdominal testicular germ cell tumour: a rare presentation of peritonitis. Br J Urol. 1998;82(4):603-604.

5. Ben-Baruch D, Yampolski I, Ziv Y. Massive haemoperitoneum complicating metastasis in the liver. Eur $\mathrm{J}$ Oncol. 1990;16(5):462-463

6. Davis JB, Schrenken JR, Zimmerman O. Massive hemoperitoneum from rupture of bening hepatocellular adenoma. Surgery. 1973;73(2):181-184.

7. Fidas-Kamini A, Busuttil A. Fatal haemoperitoneum from ruptured hepatic metastases from testicular teratomas. $\mathrm{Br}$ J Urol. 1987;60(1):80-81.

8. Bochner BH, Lerner SP, Kawachi M, Williams RD, Scardino PT, Skinner DG. Postradical orchiectomy hemorrhage: should an alteration in staging strategy for testicular cancer be considered?. Urology. 1995;46(3):408-411.

9. Cunningham LN, Ginsberg P, Manfrey S, Finkelstein LH. Massive haemorrhage secondary to metastatic testicular carcinoma. J Am Osteopath Assoc. 1989;89:341-344.

10. Motzer RJ, Bosl GJ. Hemorrhage: a complication of metastatic testicular choriocarcinoma. Urology. 1987;30(2):119122.

11. Rodier JM, Pujade-Lauraine E, Guillonneau B, Chauvenet L, Bernadou A. Haemoperitoneum due to necrosis of bulky retroperitoneal metastases: an unusual complication of chemotherapy for testicular cancer. $\mathrm{Br} \mathrm{J}$ Urol. 1996;77(6):919-920.

12. Molina Infante J, Beceiro Pedreño I, Ripoll Noiseux C, Marín Jiménez I, González Asanza C, Menchén FernándezPacheco P. Gastrointestinal hemorrhage due to metastatic choriocarcinoma with gastric and colonic involvement. Rev Esp Enferm Dig. 2004;96(1):77-80.

13. Lox CD, Trevino J. Abnormal haematological indices associated with metastatic choriocarcinoma in a young man. $\mathrm{J}$ Med. 1983;14(2):95-101.

14. Johnson DE, Appelt G, Samuels ML, Luna M. Metastases from testicular cancer. Urology. 1976;8(3):234-239.

Correspondencia autor: Dr. H. Pastor Navarro Servicio de Urología.

Complejo Hospitalario y Universitario de Albacete

Hermanos Falcó, s/n - 02006 Albacete

Tel.: 967597100

E-mail autor: hektorpn@hotmail.com

Información artículo: Nota clínica

Trabajo recibido: abril 2006

Trabajo aceptado: julio 2007 